\title{
Perceptions du risque de submersion marine par la population du littoral languedocien : contribution à l'analyse de la vulnérabilité côtière
}

\author{
Anne-Peggy Hellequin ${ }^{1}$, Hervé Flanquart ${ }^{2}$, Catherine Meur-Ferec ${ }^{3}$, Bénédicte Rulleau ${ }^{4}$ \\ 1 Géographe, Université du Littoral-Côte d'Opale, TVES EA 4477, 59383 Dunkerque, France \\ 2 Sociologue, Université du Littoral-Côte d'Opale, TVES EA 4477, 59383 Dunkerque, France \\ 3 Géographe, Université européenne de Bretagne, GÉOMER UMR 6554 CNRS LETG, IUEM, UBO, 29280 Plouzané, France \\ 4 Économiste, Université de Versailles Saint-Quentin-en-Yvelines, REEDS EA 4456, 78120 Rambouillet, France
}

Dans cet article sont présentés les résultats d'une enquête sur la perception du risque de submersion marine par les populations dans trois communes languedociennes. Il en ressort un certain paradoxe entre l'importance des dégâts que les enquêtés prévoient au niveau de l'ensemble du territoire et la relative confiance qu'ils ont pour ce qui les concerne directement. Sur ce thème encore relativement peu exploré malgré son actualité, cette contribution propose un regard original en comparant les perceptions de différentes catégories sociales comme les résidents permanents ou secondaires et les commerçants.

La Rédaction

\section{Mots-clés :}

submersion marine ; perception du risque ; vulnérabilité ; enquête par questionnaire ; Languedoc; France
Résumé - Cet article traite de vulnérabilité littorale face au risque de submersion marine. Cette vulnérabilité est divisée en quatre composantes : les aléas (phénomènes météorologiques, changement climatique, etc.), les enjeux (valorisation des biens marchands et non marchands, etc.), la gestion (politiques publiques de prévention et de réparation) et la perception des risques. Cette dernière traduit la position de chacun des différents acteurs, le degré de conscience des risques chez les habitants, leur réaction potentielle en cas de sinistre, etc. Les résultats analysés ici sont ceux d'une enquête réalisée en 2009 auprès des résidents et commerçants de trois communes du littoral languedocien. De manière générale, le risque de submersion s'avère peu redouté par les enquêtés, plutôt confiants quant à leur avenir résidentiel dans la zone. En fait, il existe une forte distorsion entre les dommages qu'ils anticipent pour l'ensemble du territoire, qu'ils jugent potentiellement très importants, et ceux qu'ils imaginent, avec beaucoup plus d'optimisme, pour leurs propres biens.

\section{Keywords:}

coastal flooding; systemic vulnerability; risk perception; questionnaire survey; France

\begin{abstract}
Risk perception of coastal flooding and coastal vulnerability: an example from Languedoc (France). Our research approach to vulnerability is based on four main components: hazards (marinemeteorological factors and climate change, geomorphological processes, etc.), assets at stake (identification and valuation, market and non market goods, damage valuation, etc.), management (public prevention and restoration policies), and perception of risk. Risk perception is a central feature in systemic vulnerability evaluation as it provides invaluable information on the diversity of stakeholder viewpoints, the degree of awareness and susceptibility of the exposed inhabitants, their attachment to the territory, their response to the hazard, etc. and thus gives indications on their adaptation capacity. Our paper analyzes the results of a wide survey of 563 inhabitants of a low and very urbanized stretch of the French Mediterranean coast (Palavas zone, Hérault) conducted in 2009, before the Xynthia storm. Results show, for example, that when respondents were asked to choose from a wide range of risks of all types, they expressed greater fears regarding non-environmental risks. When we concentrated on environmental risks and asked the respondents to choose the three they considered to be the most important for their
\end{abstract}

Auteur correspondant : A.-P. Hellequin, aph@univ-littoral.fr 
coastal area, "sea and pond pollution" was most often selected followed by "water or air pollution". The modalities related to an increase in sea level (45.5\%) and flooding by the sea or ponds (43\%) came last. This relative indifference to the danger of coastal flooding is not without consequence for home planning throughout the area considered.

Les littoraux sont le siège de nombreux processus naturels qui leur donnent une grande mobilité, parfois accentuée par l'action anthropique. Par ailleurs, l'accélération contemporaine de la remontée du niveau marin, dans un contexte de changement climatique et de pénurie sédimentaire postglaciaire, laisse prévoir l'accentuation de cette mobilité dans l'avenir. Or, lorsque celle-ci entre en résonance avec la concentration des enjeux humains à la côte, cette dynamique devient source de risques : le trait de côte tend à reculer vers l'intérieur des terres alors que les enjeux humains tendent à se rapprocher de la mer. Ces dynamiques convergentes sont à l'origine de l'émergence, puis de la prolifération, au siècle dernier, des risques liés à la mobilité du trait de côte en France. Cette tendance se retrouve sur la plupart des littoraux urbanisés du monde (Meur-Ferec et Morel, 2004). Dans un tel contexte, surtout si l'on adopte les objectifs de gestion intégrée de la zone côtière, le concept de "vulnérabilité systémique » (ou globale) nous paraît être le plus pertinent, puisqu'il intègre les éléments naturels et anthropiques dans un même système (Meur-Ferec et al., 2008). Cette approche permet de dépasser la conventionnelle « lecture manichéiste et naïve du couple aléa et vulnérabilité » (D'Ercole et Pigeon, 2000) qui dissocie aléas «naturels » et vulnérabilité "sociale ». En effet, comme l'expliquent R. D'Ercole et P. Metzger, "cette dichotomie entre aléa et vulnérabilité opère incontestablement une division du travail entre sciences « dures » qui se chargent de l'aléa et sciences sociales qui analysent la vulnérabilité, alors même que tout le monde revendique la nécessaire pluridisciplinarité pour approcher les risques» (2011, p. 6). Adopter une conception élargie et multidimensionnelle de la vulnérabilité permet au contraire d'estimer «la fragilité d'un système dans son ensemble» (D'Ercole et Pigeon, 1999). Cette approche renouvelée de la recherche sur les risques, moins "aléa centrée », est défendue depuis une quinzaine d'années par de nombreux chercheurs en sciences sociales (Becerra et Peltier, 2009 ; Gilbert, 2009) et notamment en géographie ( $D^{\prime}$ Ercole et Metzger, 2011 ; Pigeon, 2005). Cette conception systémique réunissant nature et sociétés résulte d'avancées liées à une pratique accrue de l'interdisciplinarité et à l'intégration des questions d'aménagement dans la problématique des risques dits « naturels» (Morel et al., 2006).

C'est sur ce principe, et en s'inspirant des travaux de R. D'Ercole (1994), qu'a été développée au début des années 2000, dans le cadre du Programme national d'environnement côtier (PNEC), la notion de «vulnérabilité systémique » appliquée aux risques côtiers (Meur-Ferec et al., 2003-2004). Cette vulnérabilité systémique est le fruit d'un long travail d'interdisciplinarité étendue ${ }^{1}$ (Jollivet, 1992) qui a permis l'intégration d'approches des sciences de la nature et de celles de la société autour d'un objet de recherche commun : les risques d'érosion et de submersion. Ainsi, la vulnérabilité systémique a, selon nous, quatre composantes principales : (1) les aléas (ici les phénomènes naturels, parfois influencés par l'action humaine, comme l'érosion des falaises, la rupture des cordons dunaires, la submersion, etc.), (2) les enjeux (les personnes et les biens exposés aux aléas), (3) la gestion (les politiques publiques de prévention, de protection et de gestion de crise, les équipements de défense contre la mer, etc.) et (4) la perception du risque ${ }^{2}$ (la conscience et la mémoire de celui-ci, les usages et l'attachement aux lieux exposés, la connaissance des mesures de sauvegarde, etc.).

La principale originalité de cette approche - et à notre avis aussi sa principale vertu - est qu'elle intègre les aléas dans le "système vulnérabilité » et permet ainsi une compréhension globale de la problématique des risques côtiers. En effet, si la recherche sur les aléas côtiers (modélisation des hauteurs d'eau, processus géomorphologiques, etc.) et sur les moyens de les réduire (ouvrages de protection du trait de côte, rechargement de plage, etc.) reste aujourd'hui un élément essentiel de l'étude des risques, elle ne peut suffire à la fonder à elle seule. L'examen approfondi et intégré des quatre composantes de la vulnérabilité systémique est nécessaire pour aborder la question des stratégies de gestion à long terme des côtes. Jusqu'où la mer va-t-elle monter (Paskoff, 2004) ? Comment et pour quels enjeux se défendre contre la mer? Avec quels moyens techniques? Avec quelles règles, quels financements? Quelles sont la faisabilité et l'acceptabilité sociale de l'option du repli stratégique encouragée au niveau national (Cousin, 2011) ? Les réponses sont bien sûr multiples et les choix - politiques au sens noble du terme - ne

1 Géomorphologie, géographie humaine, économie, sociologie dans le PNEC (2003-2004), puis ajouts de la physique et de la géologie dans l'ANR Miseeva (2001-2011) et enfin de la psychologie, de l'anthropologie et du droit dans l'ANR Cocorisco (2011-2014).

2 Nous utiliserons le terme de «perception du risque » car il est plus courant, notamment chez les auteurs anglo-saxons, tout en admettant sa dimension sociale et constructiviste qui en fait une représentation sociale. 
reviennent pas aux scientifiques. Cependant, il peut être utile aux décideurs de voir éclairer leurs décisions par des études montrant la contribution à la vulnérabilité globale de chacun des paramètres qui la composent (Renard et Chapon, 2010). En effet, la prévention des risques constitue, pour les élus locaux notamment, une difficulté essentielle car ils subissent de nombreuses pressions liées aux tensions sur le marché foncier et à l'impératif de développement socioéconomique de leur commune (Douvinet et al., 2011). Par ailleurs, les choix politiques de localisation des enjeux (constructions nouvelles par exemple) et de gestion des risques (mise en place des Plans de prévention des risques littoraux, attribution des permis de construire, etc.) jouent un rôle essentiel dans la production de territoires côtiers à risques et constituent un levier majeur de réduction de la vulnérabilité. Enfin, et c'est la dimension que nous développons dans cet article, la " perception » des risques par les habitants d'un territoire apparaît de plus en plus incontournable pour comprendre les logiques sociales qui lui sont propres. Bien que longtemps négligée (Peretti-Watel, 2003), elle est essentielle à la décision, car elle renseigne notamment sur les capacités d'adaptation de la société et aide à mettre en place une « bonne » gouvernance des risques ${ }^{3}$. Ainsi, des mesures de gestion techniques ou législatives visant à réduire les aléas ou les enjeux peuvent se révéler inopérantes si elles sont mal comprises et/ou mal acceptées par la population ou une partie significative de celle-ci. L'hypothèse couramment admise aujourd'hui est qu'une meilleure connaissance des risques favorise une meilleure adaptation des populations et donc une réduction de leur vulnérabilité (Giec, 2007).

Cet article présente les résultats d'une enquête par questionnaire réalisée en 2009 sur la perception du risque de submersion marine par la population de trois communes du littoral languedocien ${ }^{4}$. Il s'organise en cinq parties : la première rappellera les principaux travaux sur la perception des risques et les principaux biais qui peuvent l'affecter. Dans un second temps,

\footnotetext{
3 En France, par exemple, la tempête Xynthia a dramatiquement démontré la grande vulnérabilité des territoires côtiers en provoquant 47 morts dans la nuit du 27 au 28 février 2010, principalement sur les littoraux vendéen et charentais. En sus des problèmes d'efficacité des systèmes d'alerte, de fiabilité des digues et d'autorisation de construire en zone submersible, la question de la perception des risques par les habitants se pose avec une acuité nouvelle.

4 Cette enquête a été menée dans le cadre du projet Marine inundation hazard exposure modelling and social, economic and environnemental vulnerability assessment in regard to global change (Miseeva) financé par l'Agence nationale de la recherche à travers le programme Vulnérabilité, milieu et climat (VMC).
}

nous présenterons le contexte géographique ainsi que l'enquête mise en œuvre. Les parties suivantes s'intéresseront plus particulièrement aux résultats de cette enquête, qui mettent en évidence et analysent le degré et les formes de conscience du risque de submersion par les différentes composantes de la population des trois communes, la manière dont celles-ci se projettent à plus ou moins long terme (dans leur logement et leur commune de résidence), et les positions défendues en matière de stratégies à mettre en œuvre face à l'élévation du niveau de la mer. Nous proposerons enfin une conclusion.

\section{La perception des risques}

Percevoir un risque revient à considérer sa probabilité de survenue et l'ampleur des dégâts qu'il pourrait provoquer. Dans la démarche constructiviste qui est la nôtre (Flanquart, 2012 ; Taylor-Gooby et Zinn, 2006 ; Lupton, 1999a, 1999b), qui voit dans tout risque le produit à la fois de savoirs (connaissance des aléas et des dommages potentiels) et d'un accord social minimum sur ce qui constitue des enjeux (dommages aux biens, dommages physiques et psychologiques aux personnes ${ }^{5}$, etc.), on peut poser que la perception diffère sensiblement d'un individu à l'autre, d'un groupe social à l'autre. Chacun possède une vision différente du risque, le « regarde » à partir d'un triple point de vue : celui de son savoir à la fois scientifique et spontané, celui de ses intérêts économiques et sociaux, et celui de ses valeurs (morales, politiques, etc.). Ainsi peut-on considérer que chacun appréhende l'objet « risque » avec ce que $l^{\prime}$ 'on peut appeler des biais ${ }^{6}$, l'approche d'une façon en grande partie déterminée par sa propre position dans l'espace social et qui ne lui permet pas de le voir dans son entier. Il n'y a pas et il ne peut exister de perception exacte et neutre des risques. L'expert, par exemple, qui calcule les probabilités de survenue d'un événement, son

5 Ce n'est que depuis une trentaine d'années que, dans les pays développés, les dommages psychologiques ont été reconnus pleinement et ont pu faire l'objet d'indemnisation par les assurances lors des catastrophes et sinistres divers.

6 Le mot « biais » renvoie, ici et dans les théories exposées cidessous (celle de Kahnemann et Tversky par exemple), aux deux premiers sens qu'attribue à ce terme le Grand Robert de la langue française, tome 1 (1992) : « ligne, sens, direction oblique » et "l'un des différents côtés (d'un caractère), des différents aspects d'une chose ». La perception d'un risque par un individu - situé historiquement, géographiquement et socialement - possède en effet toujours la double particularité d'être faite d'un certain point de vue, d'un certain angle (sens 1), et d'être impuissante à englober l'ensemble de l'« objet » (sens 2). Seuls certains aspects sont perceptibles pour chacun, et encore le sont-ils de manière déformée. 
intensité probable et la valeur économique des biens potentiellement détruits et endommagés, le fait à partir des connaissances scientifiques et techniques disponibles au moment où il le fait; celles-ci faisant rarement consensus, les expertises sont donc souvent, au moins partiellement, divergentes. L'habitant du territoire à risque, que l'on nomme souvent profane et qui possède un savoir essentiellement local, peut parfois apprécier mieux que l'expert, certains aspects particuliers du risque (Wynne, 1996). Néanmoins, son approche, presque toujours parcellaire et liée à l'émotion et à ses intérêts privés, peut aussi l'éloigner de la « réalité » (si tant est que cette dernière soit objectivable) et entacher sa perception de différents biais (Horlick-Jones, 1998). Les études psychologiques, sociologiques, géographiques et anthropologiques qui analysent les perceptions des risques et tentent d'expliquer les sources de leur imprécision et de leur variabilité sont abondantes.

Ainsi plusieurs biais, qui tiennent soit aux caractéristiques des aléas, soit aux individus qui les perçoivent (identité sociospatiale), soit enfin à leur appartenance à différents groupes sociaux, peuvent être mis en évidence, sans que l'on sache toujours pourquoi et comment les expliquer.

Un premier ensemble de résultats de recherches, notamment de psychologues, sur ces biais peut être rassemblé sous le terme de "paradigme psychométrique ». Il met en évidence le fait que la perception des risques dépend en grande partie de leurs caractéristiques propres. Ainsi, selon cette approche, plus un risque est catastrophique, incontrôlable, fatal, plus il possède des conséquences de longue durée, plus il est redouté; l'archétype étant l'accident nucléaire. À l'opposé, les risques les moins redoutés sont ceux ayant des conséquences de courte durée, non fatales, contrôlables, comme l'accident de ski ou la prise d'aspirine régulière (Slovic, 1987). Des psychologues comme D. Kahnemann et al., (1988) ont aussi mis en évidence certains biais cognitifs de perception quasi universels comme celui de «facilité de représentation» qui fait que les individus ont tendance à juger comme plus fréquents (plus fort aléa) les événements qu'ils imaginent plus facilement (souvent parce que les médias en parlent plus) ou qui les impressionnent fortement (Piattelli Palmarini, 1995). Ainsi, l'accident d'avion, toujours fortement médiatisé, est-il souvent plus redouté que celui de voiture alors qu'il fait moins de morts et de blessés si on le rapporte à la distance parcourue. Autre tendance connue : les individus sont plus enclins à craindre ce que $\mathrm{N}$. Luhmann (2005) et d'autres sociologues allemands à sa suite (Hahn et al., 1994) dénomment "péril » (Gefahr en allemand), c'est-à-dire ce sur quoi ils ont l'impression de ne pas avoir de prise, qu'ils pensent subir, à la différence de ce que ces auteurs appellent «risque » : ce sur quoi nous croyons posséder un minimum de prise pour le réduire, à défaut de l'éviter totalement ${ }^{7}$. De nombreux travaux, notamment en psychologie, portent également sur ce que l'on nomme habituellement le «biais d'optimisme » (Peretti-Watel, 2003) et qui constitue une tendance très générale de l'individu à estimer que les dangers qui guettent les autres nous épargnent personnellement ou nous touchent à un degré moindre. Ce biais d'optimisme a été remarqué aussi par des auteurs d'autres disciplines (sociologie, géographie, anthropologie) même s'il est éventuellement repéré sous un autre nom. Ainsi T. Coanus et al. (2007) parlent d'un «mistigri » et de la mise en place d'une "rhétorique de dénégation du risque » chez les riverains des usines dangereuses. Il est possible de décomposer celui-ci en un " mistigri spatial » (c'est pire ailleurs) et un «mistigri plus temporel» (c'était pire avant) qui repoussent le danger, la pollution, hors du voisinage ou du temps présent (Zwarterook, 2010). Signalons enfin ce que N.D. Weinstein (1984, cité par M. Morin, 2006) définit comme « l'illusion de l'invulnérabilité ", à savoir le fait de se percevoir soi-même comme invulnérable, et qui se rapproche de ce biais d'optimisme.

Un second ensemble de travaux recouvre les nombreuses recherches menées sur les biais de perception des risques en relation avec l'appartenance des individus à différents groupes sociaux; on parlera alors de «socio-cultural perspectives » (Bickerstaff, 2004 ; Lupton, 1999b). Nous pouvons par exemple évoquer l'euphémisation des dangers éloignés dans le temps chez les personnes pauvres, qui ont des difficultés à assurer leur quotidien et se projettent donc difficilement dans le futur (Douglas et Wildavski, 1983 ; Douglas, 1992 ; Heimer, 1988 ; Lupton, 1999b ; Peretti-Watel, 2003).

Plus récemment, les recherches se sont intéressées à la «contextual risk perception » notamment chez les géographes anglo-saxons, qui insistent sur l'importance des environnements locaux pour mieux comprendre les biais de perceptions (Bickerstaff, 2004 ; Bickerstaff et al., 2006 ; Venables et al., 2009). Ainsi, les personnes interrogées ont tendance à surestimer la valeur des paysages naturels et ruraux de leur environnement proche, même s'ils sont dégradés (ou dangereux), et à se déclarer très

\footnotetext{
7 Les concepts luhmaniens de « risque » et « péril » doivent être vus comme les deux pôles opposés d'un continuum sur lequel on peut placer un risque quelconque : celui-ci se situera plus près du premier ou du second selon que l'individu confronté au danger aura ou pas l'impression de bien le contrôler. Par ailleurs, cette conviction de contrôle plus ou moins importante varie en fonction de l'identité sociale des individus. Comme le montre bien P. Peretti-Watel (2003), quand, commentant une enquête réalisée au cours des années 1990 sur la sexualité des jeunes, il explique pourquoi ceux-ci craignent plus la contamination par le virus du sida que leurs aînés : mal informés sur ses modes de transmission, ils en fantasment certains, et voient donc dans la maladie plus un péril qu'un risque.
} 


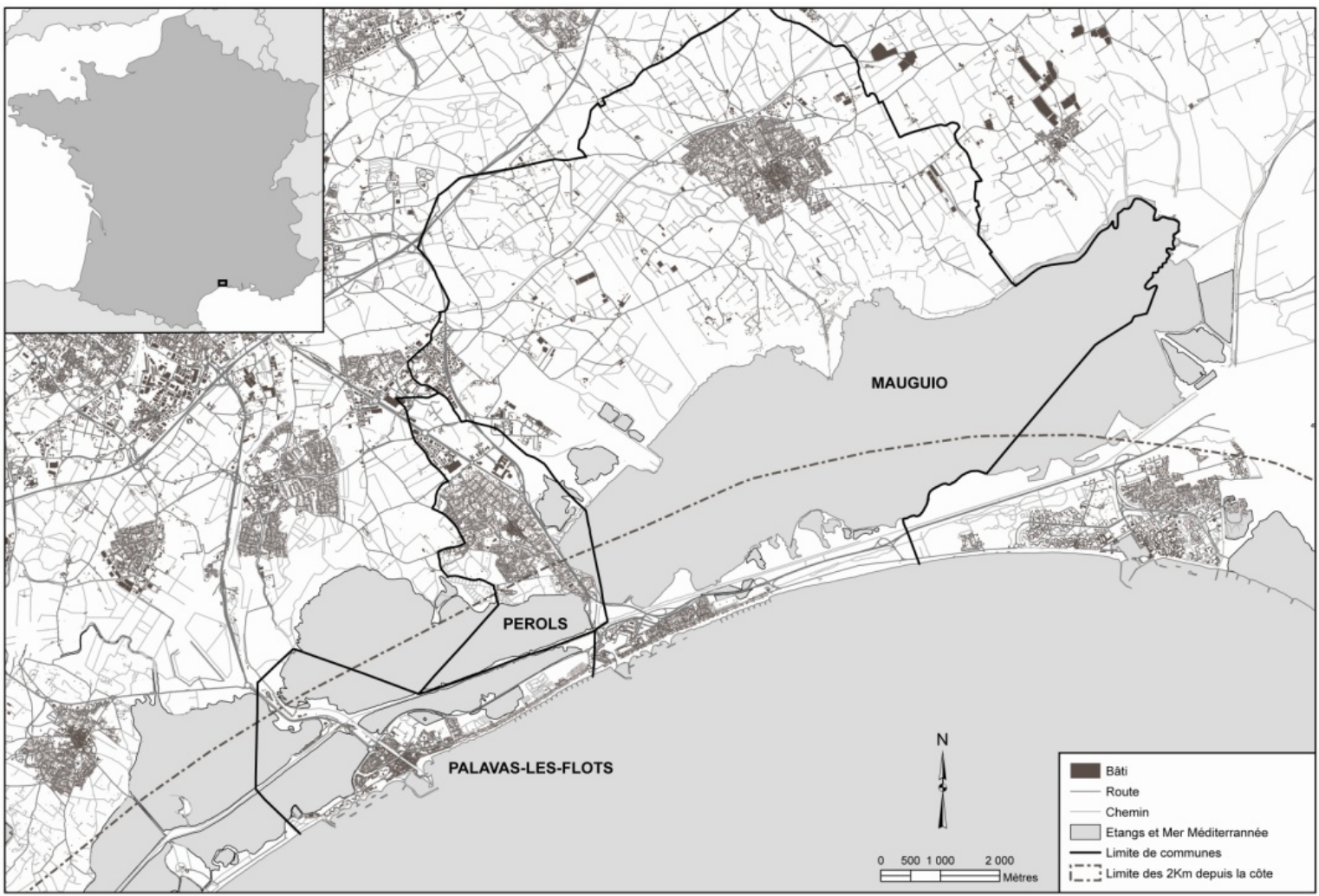

Fig. Carte de présentation du site d'enquête.

attachées à ceux-ci. Enfin, J. Tulloch et D. Lupton (2003) ont montré que la prise de risque participe de la vie quotidienne et qu'elle n'est pas forcément irrationnelle car elle semble être contrôlée personnellement. Par ailleurs, pour certaines personnes et notamment les plus jeunes, la prise de risque participe de la vie quotidienne et constitue une façon de maîtriser celle-ci et de construire son identité (Le Breton, 2002).

Si nous essayons maintenant de croiser ce bref inventaire des études sur les biais de perception des risques avec la problématique de submersion marine causée par l'accélération de la montée du niveau marin liée au changement climatique, nous pouvons émettre un certain nombre d'hypothèses. D'abord, pour le citoyen ordinaire, les dangers liés à ce phénomène devraient être particulièrement redoutés, puisqu'il n'a pas de prise directe sur eux, pas de capacités personnelles de les amoindrir, qu'ils représentent donc plus des « périls » au sens de N. Luhmann que des "risques ». Ensuite, en utilisant la typologie proposée par P. Slovic (1987) et reprise par P. Peretti-Watel et B. Hammer (2006), nous pouvons penser que le risque de submersion est également redouté des profanes car peu connu, mal évalué par les scientifiques et possédant des effets à la fois globaux et irréversibles. Si tel était le cas, on se rapprocherait alors des risques liés à l'effet de serre étudiés par ces deux derniers auteurs. À partir de ces travaux, nous pourrons ainsi mieux comprendre la conscience du risque de submersion marine, condition plus que nécessaire à une bonne acceptabilité des politiques publiques qui pourraient réduire la vulnérabilité des sociétés.

Une enquête par questionnaire auprès des résidents de la zone d'étude concernée a été menée afin de mieux comprendre la perception du risque de submersion marine.

\section{Présentation du site et méthodologie de l'enquête}

\section{La zone d'enquête et la population interrogée}

Le secteur d'enquête se situe sur la côte méditerranéenne française du département de l'Hérault. Il s'agit d'une côte très basse à morphologie de lidos (cordons sableux) et de lagunes (étangs en communication plus ou moins étroite avec la mer), particulièrement sensible au risque de submersion marine (Fig.). Cette zone d'étude regroupe plus particulièrement trois communes à dominante touristique et résidentielle : Mauguio, Palavas-lesFlots et Pérols, ayant respectivement 15 514, 5974 et 8545 habitants (Insee, 2006). 
Ces communes se caractérisent à la fois par une croissance démographique forte entre les deux derniers recensements de 1999 et 2006, et par un vieillissement de la population. La part des résidences secondaires y étant importante $^{8}$ (près de $60 \%$ à Palavas, $30 \%$ à Mauguio et un peu plus de $10 \%$ à Pérols, [Insee, 2006]), nous avons choisi d'enquêter les deux types de populations. Par ailleurs, les possesseurs de résidences secondaires jouent aujourd'hui un rôle de plus en plus important dans les politiques des communes où elles se situent; ils sont souvent très attachés à ces territoires, au point que l'on parle aujourd'hui de «bi-résidences » (ou de domiciliation «multilocale») (Bonnin et de Villanova, 1999, cité par Urbain, 2002). Enfin, la frontière entre résidents principaux et secondaires est poreuse puisqu'une part de plus en plus importante des premiers envisage de transformer son habitation de vacances en résidence principale lorsqu'elle cessera son activité professionnelle. À ces résidents principaux et secondaires, nous ajoutons également les commerçants, définissant ainsi la population-mère de notre enquête comme l'ensemble des individus qui possèdent ou utilisent régulièrement des biens ou services, marchands ou non, potentiellement exposés à l'aléa submersion marine.

Des territoires de ces trois communes, nous avons choisi de ne conserver pour l'enquête que les îlots de l'Institut national de la statistique et des études économiques (Insee) dont une partie au moins se situe à moins de deux kilomètres du trait de côte (Fig.). Nous postulons que les habitants de ces îlots, en contact proche avec la mer et les lagunes, sont potentiellement plus concernés par le risque de submersion marine.

À partir de ces choix, nous avons interrogé 563 personnes. Notre échantillon comprend dans son ensemble une part importante d'individus de plus 25 ans, de nombreux retraités ${ }^{9}$, et des personnes plutôt diplômées, puisque près $d^{\prime}$ 'un tiers de l'effectif a un niveau d'études supérieur ou égal à «bac +3 ». Dans leur ensemble, les enquêtés vivent plutôt en couple, sans enfants de moins de 16 ans à leur domicile, à l'exception des commerçants. Les cadres sont nombreux et très peu de personnes interrogées ont un métier en lien avec le littoral et/ou les

8 En moyenne, d'après le recensement de 2006, les résidences secondaires représentent $21 \%$ des logements en LanguedocRoussillon.

9 Notre échantillon de résidents secondaires est particulièrement âgé : près $42 \%$ des personnes interrogées ont plus de 65 ans. L'Insee ne s'intéressant pas aux populations des résidences secondaires dans le Recensement général de population, il n'est pas possible de savoir si cette structure par âge est représentative de celle des résidents secondaires ou si nous sommes confrontés à un biais de disponibilité, les personnes plus âgées étant plus souvent dans leur logement et donc plus disponibles pour la passation du questionnaire. risques. Concernant le logement, si seulement $17 \%$ des résidents principaux enquêtés ont une vue sur mer, plus de la moitié des résidents secondaires (54\%) en bénéficient, élément certainement important dans leur choix résidentiel.

\section{Méthodologie de l'enquête}

Pour chacune des deux populations (résidents et commerçants), un questionnaire spécifique a été construit à la croisée de la sociologie, de la géographie et de l'économie permettant ainsi de mettre en évidence les facteurs sociaux et spatiaux de la perception des risques. Pour les résidents, les thématiques abordées étaient (1) le logement (nature de l'habitat et parcours de l'enquêté en termes de résidence), (2) les attraits locaux et l'attachement au territoire, (3) les usages de la plage et des lagunes, (4) la connaissance du risque de submersion marine et le sentiment d'exposition, (5) la capacité de mobilisation $^{10}$ et (6) les caractéristiques démographiques, sociales et économiques des répondants. Ce questionnaire se composait de 158 questions, la plupart fermées. Pour les commerçants, certaines questions ont été remplacées par d'autres, relatives à leur activité et aux caractéristiques de l'entreprise (type d'activité, période d'ouverture, chiffre d'affaires, etc.). La méthode d'échantillonnage a elle aussi été fonction de la population concernée. Pour les résidents, l'approche retenue a été celle des quotas ; ces derniers ont été calculés en fonction du nombre de logements par îlot et de la répartition entre résidences principales et secondaires. En revanche, la population-mère des commerçants étant relativement réduite, une enquête à vocation exhaustive a été mise en place. Les enquêtes ont été réalisées en face à face sur site (au domicile des résidents et sur le lieu de travail des commerçants) en deux phases, la première en avril 2009, durant les vacances de printemps de la zone de Montpellier, et la seconde en juillet 2009 durant les congés d'été. Les questionnaires ont été recueillis par les auteurs de l'article lors de ces deux campagnes et par des vacataires recrutés à cette occasion. Répartis sur les îlots concernés par l'enquête, les enquêteurs sollicitaient les occupants des logements et les commerçants au fil des rues de la zone concernée, de 8 heures à 20 heures, six jours par semaine, la prise de rendez-vous ayant été minime. Les refus ont été assez nombreux, même s'ils n'ont pas été quantifiés précisément, mais plus encore c'est la faible occupation des habitations qui a le plus posé de problèmes. La forte proportion des résidences secondaires dans les communes concernées explique en

\footnotetext{
10 Des questions spécifiques ont été élaborées pour mesurer les consentements à payer pour des mesures de protection des biens (infrastructures, maisons, mais aussi plages et dunes) contre la submersion marine liée au changement climatique, mais elles ne seront pas détaillées ici.
} 
partie la difficulté de passation (en moyenne 4 questionnaires par jour pour une présence de 12 heures sur le terrain, le temps de passation étant de 30 à 40 minutes). Au final, 563 personnes ont été interrogées : 318 résidents principaux, 163 résidents secondaires et 81 commerçants. Compte tenu des imprécisions quant à la population-mère (il n'existe pas de recensement exhaustif de la population des résidences secondaires), nous n'avons pas opéré de redressement des données. Les résultats constituent donc une photographie de la perception des risques en avril et juillet 2009 des résidents principaux, secondaires et des commerçants présents à ces dates.

\section{Une population globalement peu concernée par les risques littoraux}

\section{Des risques environnementaux peu présents dans les esprits}

Avant de poser des questions sur les risques spécifiquement environnementaux, nous avons, dans une démarche dite " en entonnoir ", confronté les enquêtés à un large éventail de risques de toute nature. Les résultats de l'enquête ont montré que les craintes se portent plus vers des risques non environnementaux et individuels. Viennent en effet en premier la peur de la maladie grave (64\% la citent au moins une fois), puis de l'accident de la route $(52,5 \%)$. Il s'agit de deux risques individuels dont l'existence est rappelée chaque jour par les médias et les conversations avec l'entourage, qui donc apparaissent bien réels et dont on ne peut jamais se sentir totalement à l'abri. Si la maladie est encore plus crainte que l'accident de la route, c'est probablement parce qu'il s'agit d'un «péril » au sens de N. Luhmann. Ces craintes se retrouvent, quel que soit l'âge des personnes interrogées, même si les plus âgées ont davantage cité en premier la maladie (test du khi deux significatif à $1 \%$ ).

Trois risques environnementaux arrivent ensuite, mais loin derrière les premiers, puisque la pollution de l'eau n'est choisie en première réponse que par $9 \%$ des enquêtés (28,5\% du total des réponses), l'inondation par $7,5 \%(19,5 \%$ du total) et la pollution de l'air par $6 \%$ ( $20,5 \%$ du total). Étonnamment, pour cette population pourtant installée sur une côte basse, la crainte de l'inondation n'arrive qu'en quatrième position. Il apparaît donc d'ores et déjà que ce risque n'est pas quelque chose qui obnubile les habitants de la zone d'enquête, qui leur fasse oublier leurs autres préoccupations et inquiétudes. Il est vrai que, contrairement à l'accident de la route ou la maladie grave - voire la pollution de l'air ou de l'eau -, l'inondation/la submersion n'est que rarement associée dans $\mathrm{l}^{\prime}$ esprit des individus à un danger morte ${ }^{11}$. Notons

\footnotetext{
11 L'enquête, réalisée en 2009, est, rappelons-le, antérieure de près d'un an à la tempête Xynthia.
}

que sur ces questions d'inquiétudes générales, aucune différence n'a pu être trouvée entre les résidents principaux et secondaires (test du khi deux non significatif).

\section{La pollution généralement plus redoutée que la submersion marine}

Le questionnaire se concentrait ensuite sur les risques environnementaux et demandait aux enquêtés de choisir parmi ceux-ci, les trois qu'ils jugent les plus importants pour le littoral de leur commune.

Une pollution marine et des étangs est la modalité la plus fréquemment choisie ( $55 \%$ en fréquence cumulée), suivie par une pollution de l'eau ou de l'air (50\%). Les modalités liées à une augmentation du niveau de la mer $(45,5 \%)$ et à une inondation par la mer ou les étangs ( $43 \%$ ) ne viennent qu'ensuite et semblent plus éloignées des premières préoccupations des personnes interrogées.

Parmi les différents types de populations de la zone enquêtée (Tab. 1), nous rencontrons des différences significatives pour les réponses (test du khi deux significatif à $5 \%$ ). Alors même qu'ils ont tendance à moins choisir la modalité concernant l'augmentation générale du niveau de la mer, les commerçants surestiment en revanche les risques ponctuels de submersion de tempêtes et d'inondations que ce soit par les fleuves ou par la mer et les étangs. Il semble que cette inquiétude se développe plutôt à un horizon temporel court (celui de leurs activités commerciales, puisque $60 \%$ d'entre elles existent depuis moins de dix ans) et beaucoup moins dans le long ou très long terme (celui de la montée du niveau de la mer). À l'opposé des commerçants, les résidents secondaires sont les plus optimistes en ce qui concerne les inondations, qu'elles viennent des fleuves, de la mer ou des étangs, et pour les tempêtes. Leur présence en pointillé sur le littoral et surtout à la belle saison, le fait que leur vie quotidienne et leur activité professionnelle se déroulent ailleurs pourraient être la cause de ce qui peut être vu soit comme une connaissance tronquée de la dynamique côtière, soit comme de l'optimisme, soit encore comme un composé des deux.

Après ces questions générales sur les principales inquiétudes des enquêtés, nous nous sommes intéressés aux temporalités envisagées pour le risque d'élévation du niveau de la mer. Rappelons d'abord que les experts s'accordent à estimer la montée la plus probable du niveau marin d'ici 2100 entre $40 \mathrm{~cm}$ et 1 mètre (Giec, 2007 ; Onerc, 2009). Il est intéressant de noter que les réponses à notre questionnaire sont assez tranchées : $6 \%$ des personnes interrogées pensent que l'élévation du niveau de la mer sera peu importante et qu'il faut donc peu s'en préoccuper, et $11 \%$ doutent de la fiabilité des projections. Nous pourrions les qualifier d'optimistes. $\mathrm{Au}$ contraire, 80 \% des enquêtés pensent qu'il va falloir 
Tableau 1. Les trois risques environnementaux les plus craints pour le littoral languedocien.

\begin{tabular}{|c|c|c|c|c|}
\hline & $\begin{array}{l}\text { Résidents } \\
\text { principaux }\end{array}$ & $\begin{array}{l}\text { Résidents } \\
\text { secondaires }\end{array}$ & Commerçants & TOTAL \\
\hline $\begin{array}{l}\text { Une pollution marine } \\
\text { ou des étangs }\end{array}$ & $54 \%$ & $56 \%$ & $59 \%$ & $55 \%$ \\
\hline $\begin{array}{l}\text { Une pollution de l'air } \\
\text { ou l'eau }\end{array}$ & $51 \%$ & $50 \%$ & $46 \%$ & $50 \%$ \\
\hline $\begin{array}{l}\text { Une augmentation } \\
\text { du niveau de la mer }\end{array}$ & $47 \%$ & $46 \%$ & $40 \%$ & $46 \%$ \\
\hline $\begin{array}{l}\text { Une inondation par la mer } \\
\text { ou les étangs }\end{array}$ & $44 \%$ & $38 \%$ & $51 \%$ & $43 \%$ \\
\hline Une tempête & $27 \%$ & $25 \%$ & $35 \%$ & $27 \%$ \\
\hline $\begin{array}{l}\text { Une inondation par le Lez } \\
\text { ou les autres fleuves }\end{array}$ & $22 \%$ & $16 \%$ & $26 \%$ & $21 \%$ \\
\hline Un tsunami & $14 \%$ & $10 \%$ & $12 \%$ & $12 \%$ \\
\hline Un accident industriel & $10 \%$ & $9 \%$ & $n s$ & $9 \%$ \\
\hline Autre/ne sait pas & $n s$ & $5 \%$ & $n s$ & $2 \%$ \\
\hline Aucun & $2 \%$ & $n s$ & $n s$ & $2 \%$ \\
\hline
\end{tabular}

Question : Quels sont les trois risques environnementaux qui vous inquiètent le plus pour le littoral languedocien? Les personnes interrogées avaient le droit à plusieurs réponses.

Source : Enquête Miseeva, 2009.

envisager ce phénomène dans les années à venir, dont un peu plus de la moitié dans la décennie; les autres ne l'envisageant qu'au-delà de cet horizon de dix ans.

Paradoxalement, $73 \%$ des personnes interrogées se sentent peu ou pas exposées dans leur logement. Nous retrouvons ainsi les phénomènes connus en perception des risques, à savoir la propension à minimiser le risque et à le rejeter plus loin dans l'espace ou dans le temps (voir supra), que nous pouvons sans aucun doute rapprocher du mistigri à propos des risques industriels de T. Coanus (2007) et des travaux de F. Zonabend (1989) montrant que la distance kilométrique n'est pas le facteur le plus pertinent quant à l'appréhension du risque nucléaire puisque l'on peut se sentir proche du risque et peu exposé pour autant. L'âge joue ici un rôle très discriminant et assez différent des résultats «habituels » (Hellequin et Zwarterook, 2010 ; Zwarterook, 2010 ; Flanquart et al., 2006). En effet, les 25-45 ans ont plus tendance à envisager le risque de remontée du niveau marin dans les dix ans à venir que les plus âgés, qui au contraire sont plus nombreux à penser qu'il est surestimé (test du khi deux significatif à $1 \%$ ). Ce résultat est original car, le plus souvent, les personnes âgées se déclarent plus concernées que les plus jeunes par les risques environnementaux, la sensibilité à l'environnement s'accroissant en vieillissant (Ifen, 2003 ; Menard et al., 2008) $)^{12}$. Cette originalité tient probablement au fait que l'élévation du niveau de la mer constitue un élément

\footnotetext{
${ }^{12}$ Même si, comme le remarquaient B. Chauvin et D. Harmand (2006), le facteur âge est complexe et parfois contradictoire selon les recherches et les risques considérés.
}

de risque "socialement amplifié »(Kasperson, 2003), c'est-à-dire se trouvant au cœur d'intenses échanges sociaux (médias, discours politiques, discours savants, controverses, etc.), qui ont tendance à plus toucher les 25-45 ans à travers leur insertion professionnelle et les types de sociabilité qui leur sont propres.

\section{Une vision paradoxale de l'avenir du territoire enquêté}

\section{Des projections résidentielles très stables et confiantes dans l'avenir}

Au-delà de la plus ou moins grande « sensibilité » aux risques, nous avons fait, ainsi que nous l'avons évoqué précédemment, 1 'hypothèse que les projections résidentielles des personnes interrogées sont liées à leur perception des risques. Ainsi, l'inquiétude se traduirait par une volonté de mobilité résidentielle, certes plus ou moins aisée à mener car tous les individus ne disposent pas du même «capital résidentiel » et de la même " capacité à déménager » (Debrand et Taffin, 2005), alors que la confiance dans l'avenir se matérialiserait par une volonté de stabilité résidentielle.

Quarante-cinq pour cent des résidents principaux interrogés pensent qu'ils résideront dans le même logement dans vingt ans ${ }^{13}$ (Tab. 2). Et si nous y ajoutons ceux

\footnotetext{
${ }^{13}$ Il faut préciser que cette question leur a été posée en tout début d'enquête et qu'ils ne savaient pas à ce stade que nous nous intéressions aux risques de submersion marine.
} 
Tableau 2. Projections résidentielles des résidents principaux.

\begin{tabular}{|c|c|c|}
\hline & Fréquence & $\begin{array}{l}\text { Fréquences } \\
\text { cumulées }\end{array}$ \\
\hline Dans le même logement & $45 \%$ & \multirow{4}{*}{$\begin{array}{l}70 \% \text { dans une commune } \\
\text { littorale du L.-R. }\end{array}$} \\
\hline Dans la même commune mais dans un autre logement & $13 \%$ & \\
\hline Dans une autre commune littorale du secteur de Montpellier & $5 \%$ & \\
\hline Dans une autre commune littorale de la région Languedoc-Roussillon & $7 \%$ & \\
\hline Dans une autre commune non littorale du secteur de Montpellier & $3 \%$ & \multirow{4}{*}{$\begin{array}{l}30 \% \text { ailleurs que dans une } \\
\text { commune litt. du L.-R. }\end{array}$} \\
\hline Dans une autre commune non littorale de la région Languedoc-Roussillon & $4 \%$ & \\
\hline Ailleurs & $16 \%$ & \\
\hline NSP & $8 \%$ & \\
\hline TOTAL citations & $100 \%$ & $100 \%$ \\
\hline
\end{tabular}

Question : Où pensez-vous résider dans vingt ans ? (résidents principaux)

Source : Enquête Miseeva, 2009.

qui pensent habiter dans la même commune mais dans un autre logement ou dans une autre commune du littoral montpelliérain, voire languedocien, $70 \%$ souhaitent rester dans un environnement littoral identique ou proche.

Quant aux résidents secondaires, $72 \%$ pensent qu'ils posséderont toujours leur habitation dans vingt ans. Ces chiffres élevés marquent une projection de vie future stable géographiquement et marquée par la confiance en l'environnement immédiat. Par ailleurs, $77 \%$ des résidents principaux et secondaires pensent que la valeur marchande de leur logement va augmenter ou rester stable. Cette confiance (pourtant exprimée au printemps 2009, c'est-à-dire juste après le début de la crise économique à l'automne 2008) est également révélatrice de la non-prise en considération des éventuelles conséquences économiques des risques côtiers à cette échéance (ou peut-être de la croyance en leur prise en charge par les pouvoirs publics ${ }^{14}$ ). Enfin, cette confiance dans l'avenir sur le littoral s'accompagne d'un fort désir de transmission : $75 \%$ des résidents principaux et secondaires pensent léguer un jour leur logement à leurs enfants. Cette prévision est beaucoup plus forte chez les résidents secondaires ( $86 \%$ contre $43 \%$, test du khi deux significatif à $1 \%$ ). Tous ces chiffres montrent une projection de vie future stable sur le territoire enquêté ; celle-ci étant encore plus marquée pour les résidents secondaires que pour les principaux. Ce résultat est probablement lié à un attachement plus fort au logement secondaire qui peut constituer le point résidentiel stable au regard d'un parcours professionnel amenant à déménager souvent et représenter un lieu privilégié des relations intergénérationnelles (Luka, 2008). Nous pouvons

\footnotetext{
${ }^{14}$ En France, le système «CatNat » indemnise les victimes lorsque l'État estime qu'ils ont été victimes d'une « catastrophe naturelle ». Ce fonds est alimenté par prélèvement obligatoire (dont le taux est fixé par l'État) sur les cotisations des contrats d'assurance de tous les citoyens assurés.
}

aussi très certainement le mettre en relation avec la moins bonne connaissance des tempêtes hivernales précédemment évoquée.

L'exploitation de résultats sur les projets résidentiels ne permet donc pas de détecter des signes marquants de crainte pour l'avenir sur la côte, du moins pas suffisants pour contrebalancer les aménités que procure le littoral et pour que les habitants, résidents principaux ou secondaires, envisagent de déménager. Cette confiance en l'avenir et la faible prise en compte personnelle du risque d'élévation du niveau de la mer s'accompagnent, il est vrai, d'une très faible expérience de l'inondation, puisque seuls $10 \%$ des personnes interrogées ont déjà été inondées dans leur logement ou commerce.

\section{Mais de très forts dommages envisagés suite à une élévation du niveau de la mer}

Malgré la confiance dans leur avenir résidentiel personnel, les enquêtés envisagent des dommages importants liés aux risques de submersion marine, tant sur les espaces naturels que sur le bâti et les infrastructures. Alors qu'au début du questionnaire la submersion marine était abordée comme une modalité de risque parmi d'autres afin de ne pas biaiser les réponses des enquêtés, elle a fait ensuite l'objet de questions plus précises. Ainsi, lorsque nous leur avons demandé « quel(s) effet(s) pourrait avoir la montée du niveau de la mer en Languedoc-Roussillon », plus des trois quarts des personnes interrogées ont évoqué le recouvrement des plages et des terrains à très faible altitude. De même, $90 \%$ pensent que ce phénomène engendrera des dégâts sur le bâti et/ou le réseau routier dans les vingt ans à venir ; $45 \%$ pensent même que des quartiers entiers seront inhabitables. Pour essayer de mieux cerner les représentations du futur, nous avons posé une question ouverte sur l'avenir à long terme du lido : $42 \%$ des personnes interrogées l'imaginent fortement modifié, voire submergé. Seuls $8 \%$ prévoient que les choses resteraient 
Tableau 3. Méthodes préférées pour lutter contre l'érosion des plages.

\begin{tabular}{|l|c|}
\hline Méthode préférée & Fréquence \\
\hline Construction ou renforcement de brise-lames, digues, épis, enrochements & $41 \%$ \\
\hline Construction de barres sous-marines en sable & $24 \%$ \\
\hline Rechargement de la plage en sable & $14 \%$ \\
\hline Recul stratégique, abandon du bâti en première ligne & $11 \%$ \\
\hline Laisser-faire & $4 \%$ \\
\hline Autre méthode & $2 \%$ \\
\hline Non réponse/NSP & $5 \%$ \\
\hline TOTAL & $100 \%$ \\
\hline
\end{tabular}

Question : Pour lutter contre l'érosion de la plage on peut envisager plusieurs méthodes. Laquelle de ces méthodes préféreriez-vous voir mettre en place?

Source : Enquête Miseeva, 2009.

quasiment identiques en l'absence d'interventions particulières, et $10 \%$ imaginent que le milieu pourrait être préservé par l'action de l'homme.

Les réponses à ces questions sur les conséquences de la montée du niveau de la mer montrent que les enquêtés s'attendent à de très importants dommages dans les vingt prochaines années. Cette approche plutôt pessimiste est probablement à mettre en relation avec la forte médiatisation de la problématique du réchauffement climatique qui entraînerait une accélération de l'élévation du niveau de la mer (Fodor et Brunetière, 2011). Dans ce discours dominant, la diffusion de cartographies montrant l'avancée de la mer sur les côtes à l'aide d'images de synthèse, ainsi que l'association systématique du changement climatique à chaque événement extrême (tempête, cyclone, orage, etc.) entraînent une ambiance médiatique $^{15}$ (Joffe, 2005) dans laquelle les enquêtés s'insèrent, appliquant les images entrevues aux littoraux les plus proches.

$\mathrm{Au}$ final, ces résultats apparaissent étonnants au regard des projections personnelles dans l'avenir qui, nous l'avons vu plus haut, sont plutôt caractérisées par la stabilité résidentielle et la confiance. Ce décalage vient probablement des biais cognitifs identifiés en sociologie et psychologie sociale du risque et rappelés en début de cet article. On peut y voir à la fois la crainte d'un « péril » collectif sur lequel on n'a pas de prise, et dont les dommages prévisibles sont de plus amplifiés par sa «facilité de représentation » liée à la médiatisation du changement climatique, et la tendance à la minimisation du risque pour soi et à l'illusion de l'invulnérabilité personnelle. Ces biais influencent très certainement les comportements et les stratégies élaborées par les personnes

\footnotetext{
${ }^{15}$ Les questions de surestimation des risques (ou de sousestimation) en relation avec les médias ont été travaillées notamment dans Pidgeon et al., 2003.
}

interrogées quant à leur protection face au risque de submersion.

\section{Quelles stratégies pour l'avenir?}

Les stratégies de gestion future du trait de côte impliquent plusieurs options qui sont aujourd'hui clairement exposées, notamment dans le rapport Eurosion (European Commission, 2004) et dans le guide national de gestion du trait de côte (MEEDDM, 2010). Elles impliquent de choisir, selon les cas, entre trois orientations principales : (1) tenir la ligne (en renforçant les défenses contre la mer dans les secteurs de plus forts enjeux collectifs), (2) adopter le retrait stratégique en détruisant des bâtiments ou des infrastructures là où les enjeux sont limités, où la vie humaine est irrémédiablement menacée, (3) laisser faire la dynamique côtière là où la logique de préservation des espaces de nature l'autorise. Mais si la théorie paraît claire en matière de stratégie globale, les enquêtes auprès de la population révèlent souvent des positions individuelles plus complexes.

\section{Une préférence pour les protections « dures »}

Concernant les méthodes de lutte contre l'érosion des plages, les opinions des personnes interrogées sont assez partagées même si les protections en dur semblent favorisées par le plus grand nombre (Tab. 3).

Si $41 \%$ des résidents principaux et secondaires se prononcent en faveur des ouvrages «en dur» (briselames, enrochements, épis, etc.), 38 \% préféreraient des méthodes plus souples de rechargement en sable sur la plage ou les petits fonds, et $11 \%$ seraient favorables au repli stratégique. Cette information est particulièrement précieuse, car elle montre qu'un bon nombre restent 
Tableau 4. Choix de protection pour les biens collectifs et personnels.

\begin{tabular}{|c|c|c|c|c|c|}
\hline & $\begin{array}{l}\text { Equipements } \\
\text { collectifs }\end{array}$ & $\begin{array}{c}\text { Patrimoine culturel } \\
\text { ou historique }\end{array}$ & $\begin{array}{l}\text { Paysage } \\
\text { et nature }\end{array}$ & $\begin{array}{c}\text { Activitéséconomiques, } \\
\text { touristiques }\end{array}$ & Une maison \\
\hline $\begin{array}{l}\text { Oui, quel qu'en soit le } \\
\text { coût }\end{array}$ & $54 \%$ & $29 \%$ & $34 \%$ & $19 \%$ & $18 \%$ \\
\hline $\begin{array}{l}\text { Oui, si le coût } \\
\text { est raisonnable }\end{array}$ & $30 \%$ & $50 \%$ & $42 \%$ & $54 \%$ & $35 \%$ \\
\hline Non, c'est trop cher & $1 \%$ & $7 \%$ & $6 \%$ & $12 \%$ & $24 \%$ \\
\hline $\begin{array}{l}\text { Non, c'est inévitable } \\
\text { à long terme }\end{array}$ & $11 \%$ & $10 \%$ & $13 \%$ & $10 \%$ & $18 \%$ \\
\hline Non réponse/NSP & $4 \%$ & $4 \%$ & $5 \%$ & $5 \%$ & $5 \%$ \\
\hline TOTAL & $100 \%$ & $100 \%$ & $100 \%$ & $100 \%$ & $100 \%$ \\
\hline
\end{tabular}

Question : Pensez-vous qu'il faille se protéger collectivement contre le phénomène d'inondation?

Source : Enquête Miseeva, 2009.

encore très attachés aux méthodes traditionnelles de protection, qui du fait de leur impact visuel possèdent probablement un effet rassurant. Ces techniques sont pourtant aujourd'hui souvent remises en question, même par les services de l'État qui les ont longtemps prônées (MEEDDM, 2010). Néanmoins, le fait qu'une part presque aussi importante des enquêtés préférerait des actions par apports de sédiments permet de nuancer le propos. Cela marque peut-être une évolution vers la reconnaissance de nouvelles méthodes qui ont des impacts visuels moindres, sont plus en adéquation avec la dynamique côtière, mais qui tardent à se développer en France. Enfin, 11 \% de réponses en faveur du repli stratégique constituent un score relativement important pour une option qui suscite en général le désaccord farouche des populations locales.

Il est intéressant enfin de noter qu'il n'y a pas de relation entre les réponses à cette question et les populations ni même entre les individus se projetant sur le littoral et les autres, ou entre les hommes et les femmes (tests du khi deux non significatifs). Le choix de la méthode préférée et/ou du repli semble se faire sur des critères qui n'ont pas été encore mis au jour. Il existe au contraire un lien entre la méthode préférée et le niveau de diplômes (test du khi deux significatif à $5 \%$ ) : les personnes ayant au plus le baccalauréat sont sous-représentées chez celles choisissant les barres sous-marines en sable, mais surreprésentés dans celles privilégiant les digues. Les personnes ayant fait des études supérieures (au minimum bac +5) sont quant à elles significativement moins nombreuses à préférer le retrait. De même, l'analyse de variance montre que les individus citant le laisser-faire sont significativement plus âgés (64 ans en moyenne, contre 56 pour l'échantillon), à l'inverse de ceux choisissant les barres sous-marines en sable (49 ans en moyenne).

Au-delà du choix des méthodes préférées, il apparaît important de hiérarchiser les enjeux à protéger.

\section{Protéger en priorité les enjeux collectifs}

Nous avons demandé aux résidents s'ils pensaient nécessaire de se protéger collectivement contre la submersion marine pour différents types d'enjeux : équipements collectifs, patrimoine culturel, paysage et nature, activités économiques et touristiques et enfin habitation privée (Tab. 4). La première information qui ressort ici est la forte volonté de protection collective. De façon globale, tous types d'enjeux confondus et si nous additionnons les modalités «quel qu'en soit le coût» et «si le coût est raisonnable», près des trois quarts des personnes interrogées estiment qu'il faut les protéger collectivement, seuls $14 \%$ pensent qu'il ne faut pas intervenir car la submersion est inévitable et $10 \%$ parce que cela est trop cher. Mais une analyse plus détaillée des réponses laisse apparaître une certaine hiérarchie des enjeux. La volonté de protection collective est plus forte pour les biens collectifs comme les écoles, hôpitaux, etc. («quel qu'en soit le coût » et « si le coût est raisonnable » : 84 \%), le patrimoine bâti historique $(79 \%)$ et le patrimoine naturel $(76,5 \%)$. Les réponses sont aussi fortement positives, mais davantage corrélées au coût, pour les activités économiques (73\%). Enfin, si la protection collective des biens individuels comme une maison emporte aussi une majorité de « oui » $(52,5 \%)$, elle dépend plus fortement des coûts et, surtout, c'est le seul enjeu pour lequel une part importante des personnes interrogées répond « non » (42\%). La position de ces individus se démarque ainsi du principe qui prévaut jusqu'à présent en France selon lequel tous les assurés cotisent de la même façon au fonds "CatNat», qu'ils soient ou non exposés aux risques.

\section{Un très faible investissement dans la protection des biens personnels}

En matière de protections individuelles, 93\% des enquêtés (88\% des résidents et $100 \%$ des commerçants) 
répondent qu'ils n'ont pas personnellement construit des défenses pour se protéger et qu'ils n'ont pas pris des dispositions particulières en prévision d'une inondation marine. Il n'existe pas de différence sur cette question entre les résidents principaux et secondaires (test du khi deux non significatif). Une très faible partie de la population interrogée est donc intervenue : $5 \%$ des résidents ont fait des travaux de surélévation des parties les plus basses de leur habitation ; $7 \%$ ont pris des dispositions particulières de construction de murets, $\mathrm{d}$ 'achat de planches, d'installation de drains, de travaux d'étanchéité des portes, etc.

Si la perception du risque de submersion s'est très rarement concrétisée par des interventions matérielles sur les biens, c'est probablement parce que la nécessité ne s'en est pas fait sentir pour les résidents. En effet, $90 \%$ des résidents et commerçants n'ont pas été inondés par la mer dans leur logement au cours des dix dernières années ; lorsqu'ils ont été déjà inondés, ils sont plus nombreux à avoir fait des travaux (test du khi deux significatif à $1 \%$ ). Quand on leur demande ensuite d'envisager la nécessité d'une protection, $57 \%$ répondent que, s'il y avait véritablement besoin de se protéger contre la submersion marine, ils seraient prêts à payer pour construire des défenses afin de protéger leur maison dans les vingt prochaines années. Parmi les raisons avancées par ceux qui ont répondu négativement, nous trouvons d'abord la faible exposition au risque ( $21 \%$ choisissent la modalité «J'habite en étage/à une distance suffisante de la mer et ne pense pas être vraiment concerné(e) par ce risque »), puis l'argument que protéger l'habitation serait peine perdue, les dégâts étant de toute façon inévitables (19\%). Certaines personnes choisissant la première réponse oublient qu'une inondation des rez-de-chaussée, des caves ou des parkings souterrains, même si elle n'entraîne pas de risque mortel, serait d'un point de vue économique et de vie pratique très pénalisante. Par ailleurs, un second type de raisons est invoqué pour le refus d'entreprendre des travaux, puisque $17 \%$ des répondants estiment que les dépenses de protection doivent être prises en charge collectivement, soit par l'État soit par les collectivités territoriales ${ }^{16}$. Ce faible investissement personnel dans la protection individuelle est complexe. Il s'explique peut-être, d'une part, par les biais d'optimisme et d'illusion d'invulnérabilité personnelle, qui entrent en contradiction avec toute velléité de protection des biens individuels et, d'autre part, par l'évolution des solidarités collectives et la montée de l'individualisme. Ainsi, si les personnes interrogées sont favorables aux protections collectives pour les équipements collectifs, la question de

\footnotetext{
${ }^{16}$ Quelque $12 \%$ argumentent leur refus de faire des travaux en soulignant que c'est au propriétaire et non à eux, locataires, de payer.
}

la protection de biens privés se pose, tant en termes collectifs que du point de vue des initiatives individuelles.

\section{Conclusion}

Les résultats d'une enquête de perception des risques peuvent s'avérer particulièrement utiles lorsqu'il s'agit d'établir une stratégie à long terme pour la gestion des côtes habitées. La non-prise en considération de l'aspect « perception » risque de retarder ou même de nuire à la mise en place des politiques publiques de prévention (information, maîtrise de l'urbanisation, repli stratégique, etc.), de gestion de crise et de réparation des dommages (partage des coûts). Ainsi, l'exemple des difficultés de gestion des côtes françaises soumises à la tempête Xynthia, et notamment les mobilisations de certains habitants contre la destruction de maisons gravement exposées, est éloquent. Et la perception du risque de submersion marine par les habitants est complexe, comme le montre notre enquête menée dans trois communes littorales proches de Montpellier. En premier lieu, il convient de noter que le risque de submersion marine est peu présent dans les craintes pour l'avenir du territoire exprimées par les habitants, bien qu'ils vivent sur une côte très basse, meuble, située entre mer et étangs. Ainsi, dans la suite de cette logique, les habitants montrent une grande confiance dans la pérennité à long terme de leur résidence ou entreprise, et dans leur grande majorité envisagent leur avenir sans grand changement sur le littoral languedocien. Ils se sentent peu concernés, peu exposés individuellement à ce risque de submersion, et prennent peu de disposition pour s'en protéger. Pourtant, et de manière contradictoire avec ce qui vient d'être énoncé, la très grande majorité des habitants pensent qu'il va falloir envisager la question de l'élévation du niveau de la mer, et font une évaluation pessimiste des dommages occasionnés à leur commune et aux communes voisines en cas de submersion marine. Cette perception, que nous pouvons sans conteste qualifier de paradoxale puisqu'elle fait des habitants des pessimistes lorsqu'ils envisagent l'ensemble du territoire et des optimistes lorsqu'ils se limitent à leur environnement très proche, relève bien d'un biais d'optimisme. Le même qui fait considérer à la grande majorité des conducteurs que la route est globalement dangereuse, mais que la probabilité pour qu'ils aient eux-mêmes un accident est très faible. Cette quasi-illusion de l'invulnérabilité se traduit, comme l'énonce $M$. Morin, par « une surestimation des moments de vie positifs", au détriment d'une gestion adaptée du risque (2006, p.171).

Ce biais d'optimisme au sujet des risques de submersion est d'autant plus remarquable que, contrairement à la conduite automobile, il concerne plus ce que N. Luhmann appelle un péril qu'un risque. En effet, si 
les conducteurs peuvent agir personnellement pour limiter le danger encouru en se montrant prudents, les habitants de la zone enquêtée ne peuvent avoir de vraies actions concrètes et personnelles pour se protéger et protéger leurs biens d'une remontée future du niveau marin si celle-ci était vraiment importante. Notre enquête montre d'ailleurs qu'ils sont très peu nombreux à investir dans la protection de leur résidence et de leurs biens personnels.

Si nous affinons les résultats de l'enquête, nous constatons que le type d'occupation - principal ou secondaire - de la résidence possède un pouvoir explicatif fort en matière de projets d'avenir sur cet espace et d'estimation du risque de submersion. Dans l'ensemble, les résidents secondaires possèdent une vision plus optimiste de l'avenir du littoral et s'y projettent plus dans la durée, ces différences surpassant les clivages générationnels traditionnellement observés en perception du risque. Ce biais d'optimisme plus important chez les résidents secondaires peut en partie s'expliquer par leur moins bonne connaissance du terrain et des phénomènes météo-marins, car ils ne sont généralement pas présents l'hiver, saison qui voit les tempêtes les plus importantes attaquer le littoral. L'attachement plus fort des résidents secondaires aux lieux semble également jouer à travers le phénomène d'euphémisation du risque (Peretti-Watel, 2003), voire de déni, réponse classique des individus soumis à ce que les psychologues appellent depuis L. Festinger (1957) une « dissonance cognitive » (ici entre la volonté de continuer à habiter sur le territoire et le flux croissant d'informations reçues indiquant que les risques augmentent). Notre enquête paraît enfin contredire la thèse de $P$. Slovic, puisque face à un phénomène qui peut avoir des effets importants (voire irréversibles), devant lequel l'individu isolé est impuissant et qui est encore mal évalué par les experts, l'inquiétude est, contrairement à ce qui était attendu, faible (et les recherches d'informations spontanées quasi inexistantes).

Ainsi, la perception du risque de submersion marine telle qu'elle nous est présentée à travers cette enquête apparaît complexe et pour le moins éloignée des «dires » d'experts. Le risque de submersion et les mesures de réduction de celui-ci semblent en effet peu appréhendés par les personnes interrogées. Ce décalage peut peser sur la mise en œuvre des politiques de prévention et de gestion de l'élévation du niveau de la mer et accroître de manière significative la vulnérabilité systémique des littoraux languedociens.

\section{Remerciements}

Les auteurs remercient l'ensemble de l'équipe du projet Marine inundation hazard exposure and social, economic and environmental vulnerability. Assessment in regard to global change (Miseeva) financé par l'Agence nationale de la recherche (programme Vulnérabilité, milieux et climat) pour leurs remarques, et plus particulièrement les personnes qui ont participé au recueil des données sur le terrain. Ils tiennent également à remercier les relecteurs anonymes pour leurs commentaires utiles et constructifs.

\section{Références}

Becerra, S., Peltier A., 2009. Risques et environnement : recherches interdisciplinaires sur la vulnérabilité des sociétés, Paris, L'Harmattan.

Bickerstaff, K., 2004. Risk perception research: socio-cultural perspectives on the public experience of air pollution, Environment International, 30, 827-840.

Bickerstaff, K., Simmons, P., Pidgeon, P., 2006. Situating local experience of risk: Peripherality, marginality and place identity in the UK foot and mouth disease crisis, Geoforum, 37, 844-858.

Bonnin, P., de Villanova, R., (Eds) 1999. D'une maison l'autre. Parcours et mobilités résidentielles, Grane, Éditions Créaphis.

Coanus, T., Duchêne, F., Martinais, E., 2007. L'industrie chimique et ses riverains : une relation ambivalente, Responsabilité et Environnement, 48, 68-76.

Cousin, A., 2011. Propositions pour une stratégie nationale de gestion du trait de côte, du recul stratégique et de la défense contre la mer, partagée entre l'État et les collectivités territoriales. Rapport au gouvernement.

D’Ercole, R., 1994. Les vulnérabilités des sociétés et des espaces urbains, concepts, typologie, mode d'analyse, Revue de Géographie Alpine, 4, Numéro spécial «Croissance urbaine et risques naturels dans les montagnes des pays en voie de développement », 87-96.

D'Ercole, R., Metzger, P., 2011. Les risques en milieu urbain : éléments de réflexion, EchoGéo [En ligne], 18 (http:// echogeo.revues.org/12640).

D'Ercole, R., Pigeon, P., 1999. L'expertise internationale des risques dits naturels: intérêt géographique, Annales de Géographie, 608, 339-357.

D’Ercole, R., Pigeon, P., 2000. L'évaluation du risque à l'échelle internationale : méthodologie et application aux diagnostiques préalables aux actions de préparation et de prévention des catastrophes, Cahiers Savoisiens de Géographie, 1, 29-36.

Debrand, T., Taffin, C., 2005. Les facteurs structurels et conjoncturels de la mobilité résidentielle depuis 20 ans, Economie et Statistique, 381-382, 125-146.

Douglas, M., Wildavski, A., 1983. Risk and Culture: An Essay on the Selection of Technological and Environmental Dangers, Berkeley (CA), University of California Press.

Douglas, M., 1992. Risk and Blame: Essays in cultural theory, London, Routledge.

Douvinet, J., Anselle, A., Delfossez, S., Denolle, A.-S., 2011. Les maires face aux plans de prévention du risque inondation (PPRI), L'Espace Géographique, 1, 31-46.

European Commission, 2004. Living with coastal erosion in Europe: Sediment and space for sustainability. Official UE publication office, Luxembourg. 
Festinger, L., 1957. A Theory of Cognitive Dissonance, Stanford (CA), Stanford University Press.

Flanquart, H., 2012. An unwelcome user?: Or how to negociate the use of a risky space, Journal of Risk Research, 15, 10, 12611279.

Flanquart, H., Hellequin, A.-P., Deldreve, V., Deboudt, P., 2006. Identité sociale et perception du risque côtier, in Colloque international Interactions Nature et Société: analyses et modèles, La Baule, 3-6 mai.

Fodor, F., Brunetière, V., 2011. Climat d'angoisse : l'imaginaire du changement climatique, Coll. Sciences humaines, Paris, Éditions Les 2 Encres.

Giec (Groupe intergouvernemental sur l'évolution du climat), 2007. Rapport de synthèse.

Gilbert, C., 2009. La vulnérabilité, une notion vulnérable ?, in Becerra, S., Peltier, A. (Eds.), Risques et environnement : recherches interdisciplinaires sur la vulnérabilité des sociétés, Paris, L'Harmattan.

Hahn, A., Eirmbter, W.H., Jacob, R., 1994. Sida : savoir ordinaire et insécurité, Actes de la Recherche en Sciences Sociales, 104, 81-89.

Heimer, C.A., 1988. Social structure, psychology, and the estimation of risk, Annual Review of Sociology, 14, 491-519.

Hellequin, A.-P., Zwarterook, I., 2010. Mauvais air : une pollution au quotidien, in $2^{\mathrm{e}}$ colloque international Le risque industriel, une question de sciences humaines et sociales, ENS LSH, ENTP, Lyon, 24-25 mars.

Horlick-Jones, T., 1998. Meaning and contextualisation in risk assessment, Reliability Engineering and System Safety, 59, 1, 79-89.

Ifen, 2003. Les Français et l'environnement : opinions et attitudes au début 2002, Études et Travaux, 39.

Insee, 2006. Recensement de la population.

Joffe, H., 2005. De la perception à la représentation du risque : le rôle des médias, Hermès, 41, 121-129.

Jollivet, M. (Ed.), 1992. Sciences de la nature, sciences de la société : les passeurs de frontières, Paris, CNRS Éditions.

Kahnemann, D., Slovic, P., Tversky, A., 1988. Judgement under Incertainty: Heuristics and Biases, Cambridge (UK), Cambridge University Press.

Kasperson, R.E., 2003. The social amplification of risk: Assessing fifteen years of research and theory, in Pidgeon, N., Kasperson, R.E., Slovic, P., The Social Amplification of Risk, Cambridge (UK), Cambridge University of Press, 13-46.

Le Breton, D., 2002. Conduites à risques, Paris, Puf.

Luhmann, N., 2005. Risk: A Sociological Theory, London, Aldine Transaction.

Luka, N., 2008. Le « cottage » comme pratique intergénérationnelle: narrations de la vie familiale dans les résidences secondaires du centre de l'Ontario, Enfances, Familles, Générations, 8, 86-117.

Lupton, D., 1999a. Introduction: risk and sociocultural theory, in Lupton, D. (Ed.), Risk and Sociocultural Theory, Cambridge (UK), Cambridge University Press, 1-11.

Lupton, D., 1999b. Risk, London and New York, Routledge.

Menard, C., Girard, D., Léon, C., Beck, F. (Eds), 2008. Baromètre santé environnement 2007, Coll. Baromètre santé, SaintDenis, INPES.
Meur-Ferec, C., 2008. De la dynamique naturelle à la gestion intégrée de l'espace littoral: un itinéraire de géographe, Coll. Universitaire, Paris, Édilivre.

Meur-Ferec, C., Deboudt, P., Deldrève, V., Flanquart, H., Hellequin, A.-P., Herbert, V., Morel, V., 2003-2004. La vulnérabilité des territoires côtiers : évaluation, enjeux et politiques publiques, Programme national environnement côtier (PNEC).

Meur-Ferec, C., Deboudt, P., Morel, V., 2008. Coastal risks in France: An integrated method for evaluating vulnerability, Journal of Coastal Research, 2, 178-189.

Meur-Ferec, C., Morel, V., 2004. L'érosion sur la frange côtière : un exemple de gestion des risques, Natures Sciences Sociétés, 12, 263-273.

MEEDDM (Ministère de l'Écologie, de l'Énergie, du Développement durable et de la Mer), 2010. La gestion du trait de côte, Paris, Éditions Quae.

Morel, V., Deboudt, P., Hellequin, A-P., Herbert, V., MeurFerec, C., 2006. Regard rétrospectif sur l'étude des risques en géographie à partir des publications universitaires (1980-2004), L'Information Géographique, 1, 6-24.

Morin, M., 2006. Pour une approche psycho-socio-environnementale des risques sanitaires, in Weiss, K., Marchand, D. (Eds), Psychologie sociale de l'environnement, Rennes, Presses Universitaires de Rennes, 165-177.

Onerc (Observatoire national sur les effets du réchauffement climatique), 2009. Changement climatique: coûts des impacts et pistes d'adaptation. Troisième rapport de l'Onerc.

Paskoff, R., 2004. Jusqu'où la mer va-t-elle monter ?, Les Petites Pommes du Savoir, 53, Éditions le Pommier.

Peretti-Watel, P., 2003. La Sociologie du risque, Paris, Armand Colin.

Peretti-Watel, P., Hammer B., 2006. Les représentations profanes de l'effet de serre, Natures Sciences Sociétés, 14, 4, 353364.

Piattelli Palmarini, M., [1993] 1995. La réforme du jugement ou comment ne plus se tromper, Paris, Odile Jacob.

Pidgeon, N., Kasperson, R.E., Slovic, P., 2003. The Social Amplification of Risk, Cambridge (UK), Cambridge University of Press.

Pigeon, P., 2005. Géographie critique des risques, Paris, Economica, Anthropos.

Renard, F., Chapon, P.-M., 2010. Une méthode d'évaluation de la vulnérabilité urbaine appliquée à l'agglomération lyonnaise, L'Espace Géographique, 39, 1, 35-50.

Slovic, P., 1987. Perception of risk, Science, 4799, 280-285.

Taylor-Gooby, P., Zinn, J.O., 2006. Current directions in risk research: new development in psychology and sociology, Risk Analysis, 26, 2, 397-411.

Tulloch, J., Lupton D., 2003. Risk and Everyday Life, London, Sage Publications.

Urbain, J.-D., 2002, Le résident secondaire, un touriste à part ?, Ethnologie Française, 32, 3, 515-520.

Venables, D., Pidgeon, N., Simmons, Henwood, K., Parkhill, K., 2009. Living with nuclear power: A Q-method study of local community perceptions, Risk Analysis, 29, 8, 1089-1104.

Weinstein, N. D., 1984. Why it won't happen to me: perception of risk factors and illness susceptibility, Health Psychology, 3, 431-457. 
Wynne, B., 1996. Controverses, indétermination et contrôle social de la technologie. Leçons du nucléaire et de quelques autres cas au Royaume-Uni, in Godard, O. (Ed.), Le Principe de précaution dans la conduite des affaires humaines, Paris, MSH, Inra, 149-179.

Zonabend, F., 1989. La Presqu'île au nucléaire, Paris, Odile Jacob.
Zwarterook, I., 2010. Les risques et pollutions industriels sur le territoire dunkerquois : des perceptions à la concertation, Cahiers de la Sécurité Industrielle, 2010-07 (http://www. foncsi.org/fr/publications/collections/cahiers-securiteindustrielle/concertation-Dunkerque/CSI-concertationDunkerque.pdf).

Reçu le 16 juillet 2011. Accepté le 5 décembre 2012. 